\title{
The relative impact of evolving pleiotropy and mutational correlation on trait divergence.
}

\author{
Jobran Chebib and Frédéric Guillaume \\ Department of Evolutionary Biology and Environmental Studies, University of Zürich, \\ Winterthurerstrasse 190, CH-8057 Zürich, Switzerland. email: \\ frederic.guillaume@ieu.uzh.ch
}

\begin{abstract}
Both pleiotropic connectivity and mutational correlations can restrict the divergence of traits under directional selection, but it is unknown which is more important in trait evolution. In order to address this question, we create a model that permits within-population variation in both pleiotropic connectivity and mutational correlation, and compare their relative importance to trait evolution. Specifically, we developed an individual-based, stochastic model where mutations can affect whether a locus affects a trait and the extent of mutational correlations in a population, under the corridor selection model (some traits under directional selection and others under stabilizing selection). We find that traits can diverge whether there is evolution in pleiotropic connectivity or mutational correlation but when both can evolve then evolution in pleiotropic connectivity is more likely to allow for divergence to occur. The most common genotype found in this case is characterized by having one locus that maintains connectivity to all traits and another that loses connectivity to the traits under stabilizing selection (subfunctionalization). This genotype is favoured because it allows the subfunctionalized locus to accumulate greater effect size alleles, contributing to increasingly divergent trait values in the traits under directional selection without changing the trait values of the other traits (genetic modularization). These results provide evidence that partial subfunctionalization of pleiotropic loci may be
\end{abstract}


a common mechanism of trait divergence under regimes of corridor selection.

Keywords: Pleiotropy, Correlated Mutation, Genetic Architecture, Correlational Selection, Modularity, Mutation

\section{Introduction}

One of the central problems in evolutionary biology is understanding the processes through which new traits arise. One process that can lead to the creation of new traits is when existing traits become differentiated from one another because they are selected for a new purpose (Rueffler et al., 2012). There has long been evidence that this occurs through gene duplication followed by trait divergence (Muller, 1936; Ohno, 1970; Rastogi and Liberles, 2005). One example in vertebrates is the differentiation of forelimbs from hind limbs, where the same gene that was responsible for both fore and hind limb identity in development diverged (Graham and McGonnell, 1999; Minguillon et al., 2009; Petit et al., 2017). In this case, the paralogous genes Tbx4/Tbx5 that encode transcription factors for fore/hindlimb identity likely evolved from the same ancestral gene, and their expression differentiated after duplication (Minguillon et al., 2009). Somehow during functional divergence, there was break in the constraints of genetic architecture that determined the variational ability of the traits to respond to selection as independent modules (Wagner and Altenberg, 1996; Hansen, 2006).

Where do genetic correlations and modularity in traits come from on a molecular level?

Modular structure in phenotypic covariations are found in a wide range of organisms, including yeast, round worms and mice (Wang et al., 2010). The underlying genetic architectures producing these covariations between traits are beginning to become more clear, but are still not certain. Constraints may stem from pleiotropic connections between loci and traits, where they may or may not create genetic covariation between the traits (Baatz and 
Wagner, 1997; Kenney-Hunt et al., 2008; Smith, 2016). The pleiotropic effect on constraints comes in two forms: a multi-trait genic effect or a multi-trait mutational effect (Stern, 2000). A multi-trait genic effect is when a gene product (e.g. enzyme, transcription factor, etc.) has an effect on more than one trait by having multiple substrates or binding sites. This may constrain the evolution of separate traits (even without creating genetic correlations (Wagner, 1989; Baatz and Wagner, 1997)) because the overall fitness effect of a mutation beneficial for one trait will have a larger deleterious effect proportional to the number of traits it affects (Orr, 2000; Welch and Waxman, 2003). A multi-trait mutational effect is when a single mutation induces a change in more than one trait. Of course, pleiotropic mutations are dependent on the gene being pleiotropic, but correlations in mutational effects create genetic correlations which can constrain trait divergence in addition to the constraints caused by pleiotropic genic effects (Lande, 1979; Arnold, 1992; Stern, 2000). Both types of pleiotropic effects can evolve as a result of directional selection to allow for the differentiation of traits, but it is not known which is most important in constraining or facilitating evolution (Chebib and Guillaume, 2017)

\section{Previous models of selection on pleiotropic constraints}

When there is variation in the pleiotropic effects of genes on traits or the extent of correlation in mutational effects in a population, they can both evolve in response to selection (Jones et al., 2007; Pavlicev et al., 2011; Jones et al., 2014; Melo and Marroig, 2015). Previous multi-trait models of evolution looked at the effect of selection on genetic architecture evolution, and their predictions are all dependent on the details of selection (Melo et al., 2016). Pavlicev et al. (2011) developed a deterministic model for evolution of pleiotropic gene effects under selection where polymorphic loci (rQTLs) could affect the strength of correlation between traits. Selection on all traits in the same direction favoured alleles that increased the correlations between traits. Whereas, when there was directional selection on one trait and sta- 
bilizing selection on another (called the corridor model of selection), alleles that lowered correlations between traits were favoured by evolution. Melo and Marroig (2015) developed an individual-based, stochastic model where pleiotropic connections between 500 additive loci and ten traits were variable and mutable. They found similar results as directional selection on all traits in a module led to increased correlations between traits and under the corridor model of selection, traits diverging from another became separate modules in their genetic correlations. But neither of these models explicitly allowed correlational mutations to evolve. Another group of researchers allowed mutational correlation between two traits to evolve in simulation by allowing it to be determined by ten unlinked, additive loci (Jones et al., 2003, 2007). They found that patterns of mutational correlation evolved to match correlational selection but only investigated stabilizing selection without divergence between traits, and also did not explicitly allow for pleiotropic connections to evolve.

Both pleiotropic connections (genic effects) and mutational pleiotropy (mutational effects) affect the ability of traits to diverge from one another. Whether pleiotropic connectivity or mutational correlation is more important remains to be seen (Chebib and Guillaume, 2017). Here we attempt to answer this question using stochastic simulations of populations, where individuals can vary in both pleiotropic connections and mutational effects, and divergent selection on some traits but not others will lead to divergent trait evolution.

\section{Methods}

\section{Simulation development}

We modified the individual-based, forward-in-time, population genetics simulation software Nemo (v2.3.4) to allow for the evolution of pleiotropic connectivity and mutational correlations (Guillaume and Rougemont, 2006). This was done by implementing the capability for the pleiotropic connections between alleles and traits in diploid individuals to be removed or added 
at a rate given by the pleiotropic mutation rate $\left(\mu_{\text {pleio }}\right)$, which determined whether a trait-specific allelic effect of a locus was added to a trait value in that individual (Figure 1A). Each individual had a separate set of loci that held two allelic values for each pairwise mutational correlation between traits and which mutated at a rate given by the pleiotropic mutation rate $\left(\mu_{\text {mutcor }}\right)$ (Figure 1B). Whenever a mutation occurred at the genic effect level, at a rate given by the genic mutation rate $(\mu)$, the two alleles of each mutational correlation locus were averaged and the set of correlations were combined with equal mutational variances for all traits $\left(\alpha^{2}=0.1\right)$ to make up the $\mathbf{M}$ matrix that gave the parameter values for random sampling of mutational effects from a multivariate Gaussian distribution. Mutational effects were then added to the existing allelic values (continuum-of-alleles model; Crow and Kimura, 1964). Since each individual carried its own alleles for the genic effects on traits, the pleiotropic connectivity of genic alleles to traits, and the mutational correlations of effects on genic effects, combined with mutability on all three, there was the capacity of creating variation in the population on which differential selection could act.

\section{Experimental design}

To understand the impact of directional selection on the structure of genetic architecture, simulations were run with a population of individuals that had two additive loci underlying four traits. The initial conditions were highly constrained to divergent evolution with full pleiotropy (each locus affecting every trait) and strong mutational correlations between trait pairs $\left(\rho_{\mu}=0.99\right)$. Traits all began with a value of 2 with each allele of each locus having a value of 0.5 since trait values were calculated for each trait by summing the allelic values of all loci affecting that trait (2 alleles $\mathrm{x} 2$ loci $\mathrm{x} 0.5=2$ ). Gaussian stabilizing selection was applied and determined the survival probability of juveniles, whose fitness was calculated as $w=\exp \left[-\frac{1}{2}\left((\mathbf{z}-\theta)^{\mathrm{T}} \cdot \mathbf{\Omega}^{-1} \cdot(\mathbf{z}-\theta)\right)\right]$, where $\mathbf{z}$ is the individual trait value vector, $\theta$ is the vector of local optimal trait values (all values initialized at 2 ), 

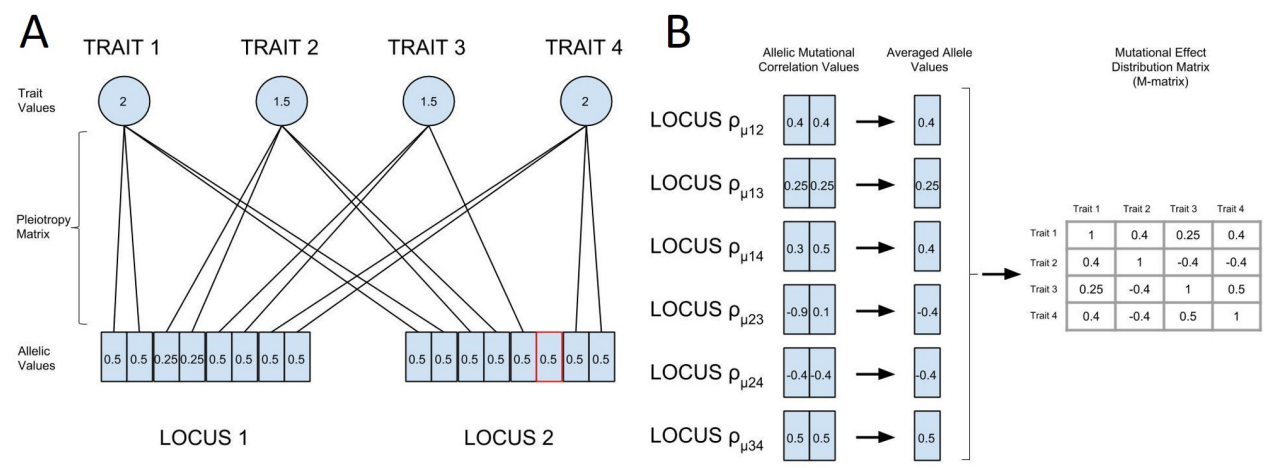

Figure 1: Pictorial representation of simulation model. A - Genetic architecture with an example of allelic values (genotype) of 2 loci, trait values (phenotype) of 4 traits, and the pleiotropic connectivity between them. Here, Trait 1 (and Trait 4) has a value of 2 determined by the sum of allelic values connected to it from Locus $1(0.5+0.5)$ and Locus $2(0.5+0.5)$. Traits 2 and 3 have the same value of 1.5 but for different reasons. For Trait 2, both alleles of Locus 1 have a value of 0.25 and both alleles of Locus 2 have a value of $0.5(0.25+0.25+0.5+0.5=1.5)$, whereas for Trait 3 both alleles of Locus 1 are connected to it but only one allele of Locus $2(0.5+0.5+0.5+0=1.5)$. B - An example of the creation of the mutation matrix (M-matrix) used for random sampling multivariate mutational effects with 6 loci representing each pairwise combination between 4 traits in an individual. Each locus has 2 alleles whose values represent correlations in mutational effects between traits that are averaged together and combined with the averages of the other mutational correlation loci into the off-diagonal elements of the $\mathbf{M}$-matrix.

and $\Omega$ is the selection variance-covariance matrix $(n \times n$, for $n$ traits) describing the multivariate Gaussian selection surface. The $\Omega$ matrix was set as a diagonal matrix with diagonal elements $\omega^{2}=5$ (strength of selection scales inversely with $\omega^{2}$ ), and off-diagonal elements set for modular correlational selection with no correlation between modules $\left(\rho_{\omega 13}=\rho_{\omega 14}=\rho_{\omega 23}=\rho_{\omega 24}=0\right)$ and strong correlation within modules $\left(\rho_{\omega 12}=\rho_{\omega 34}=0.9\right)$. Divergent directional selection proceeded by maintaining static optimal trait values for traits 3 and $4\left(\theta_{3}=\theta_{4}=2\right)$ and increasing the optimal trait values for traits 1 and 2 by 0.001 per generation (corridor model of selection sensu Wagner (1984); Bürger (1986)).

In order to compare the differential effects of evolving pleiotropic connections and evolving mutational correlations on trait divergence, nine different 
simulations were run with all combinations of three different rates of mutation in pleiotropic connections and mutational correlations ( $\mu_{\text {pleio }}$ and $\mu_{\text {mutcor }}$ $=0,0.001$ or 0.01$)$ representing no evolution, and mutation rates below, at and above the allelic mutation rate $(\mu=0.001)$, respectively. Simulations were also run with initial mutational correlations between all pairs set to 0 $\left(\rho_{\mu}=0\right)$ to compare highly constrained genetic architecture to ones with no constraints in the direction of mutational effects.

Unless otherwise specified, each simulation was run with 500 initially monomorphic (variation is introduced through mutations) individuals for 5000 generations of divergent, directional selection on traits 1 and 2 (followed by 5000 generations of stabilizing selection) in order to observe general patterns of average trait value divergence, population fitness, genetic correlation, pleiotropic degree and mutational correlation. In the case of pleiotropic degree, the two loci affecting trait values were sorted into a high and low pleiotropic degree locus for each individual before average over populations or replicates so that differential effects of the two loci were not averaged out in the final analysis. Individuals were hermaphrodites mating at random within a population, with non-overlapping generations. Statistics were averaged over 50 replicate simulations of each particular set of parameter values.

\section{Results}

\section{Trait divergence and genetic modularity under genetic constraints}

In the absence of genetic architecture evolution $\left(\mu_{\text {pleio }}=\mu_{\text {mutcor }}=0\right)$, trait modules are still capable of divergence, but do not follow trait optima closely since traits 3 and 4 get pulled away from their optima as traits 1 and 2 increase to follow theirs (Figure 2). With the introduction of variation in genetic architecture through mutation $\left(\mu_{\text {pleio }}=\mu_{\text {mutcor }}>0\right)$, average trait values follow their optima more closely and the capability of trait divergence increases as mutation rates in genetic architecture increases, which 
leads to higher average populations fitness values by generation 5000 (Figure 3). Also by generation 5000, simulations with higher genetic architecture mutation rates $\left(\mu_{\text {pleio }} \geq 0.001\right.$ or $\left.\mu_{\text {mutcor }}=0.01\right)$ have distinctly modular genetic correlation structures with stronger correlations between traits 1 and 2 than between traits 3 and 4 (Figure 4). But at the highest pleiotropic connection mutation rate $\left(\mu_{\text {pleio }}=0.01\right)$ the genetic integration of trait 3 with 4 (and even trait 1 with 2) is no longer as strong. An increase in pleiotropic connection evolution has a greater effect on trait divergence and genetic modularization in a population when compared to the same increase in mutational correlation evolution, which is evident when either $\mu_{\text {pleio }}$ or $\mu_{\text {mutcor }}$ is the same as the allelic mutation rate $(\mu=0.001)$. Even when the mutational correlation mutation rate is at the highest tested $\left(\mu_{\text {mutcor }}=0.01\right)$, an increase in the pleiotropic connectivity mutation rate still improves the ability for traits to diverge, which can be seen in the decrease in variance over replicate simulations (Figure 2).

Effects of pleiotropic connectivity and mutational correlation evolution on rate and extent of divergent evolution

When evolution of pleiotropic connections is possible $\left(\mu_{\text {pleio }}>0\right)$ the most common allele in almost all cases is one that maintains connections to traits 1 and 2, but has lost connections to traits 3 and 4 after two mutational events. This allele is found in locus 1 or 2 at a frequency of 0.873 averaged over the populations of all simulations where evolution of pleiotropic connections is possible. The allele goes to fixation or near fixation in one locus where its pleiotropic degree decreases from four to two, and this happens more rapidly as $\mu_{\text {pleio }}$ increases (Figure 5). The decrease in pleiotropic degree resulting from the increase in frequency of this allele coincides with the modularization of genetic correlations, the divergence of traits and the increase in fitness. The proportion of times in which this particular allele becomes common in locus 1 or in locus 2 is approximately equal over all simulations (0.491 and 0.509 , respectively, over 300 simulations) and never is observed in both loci 

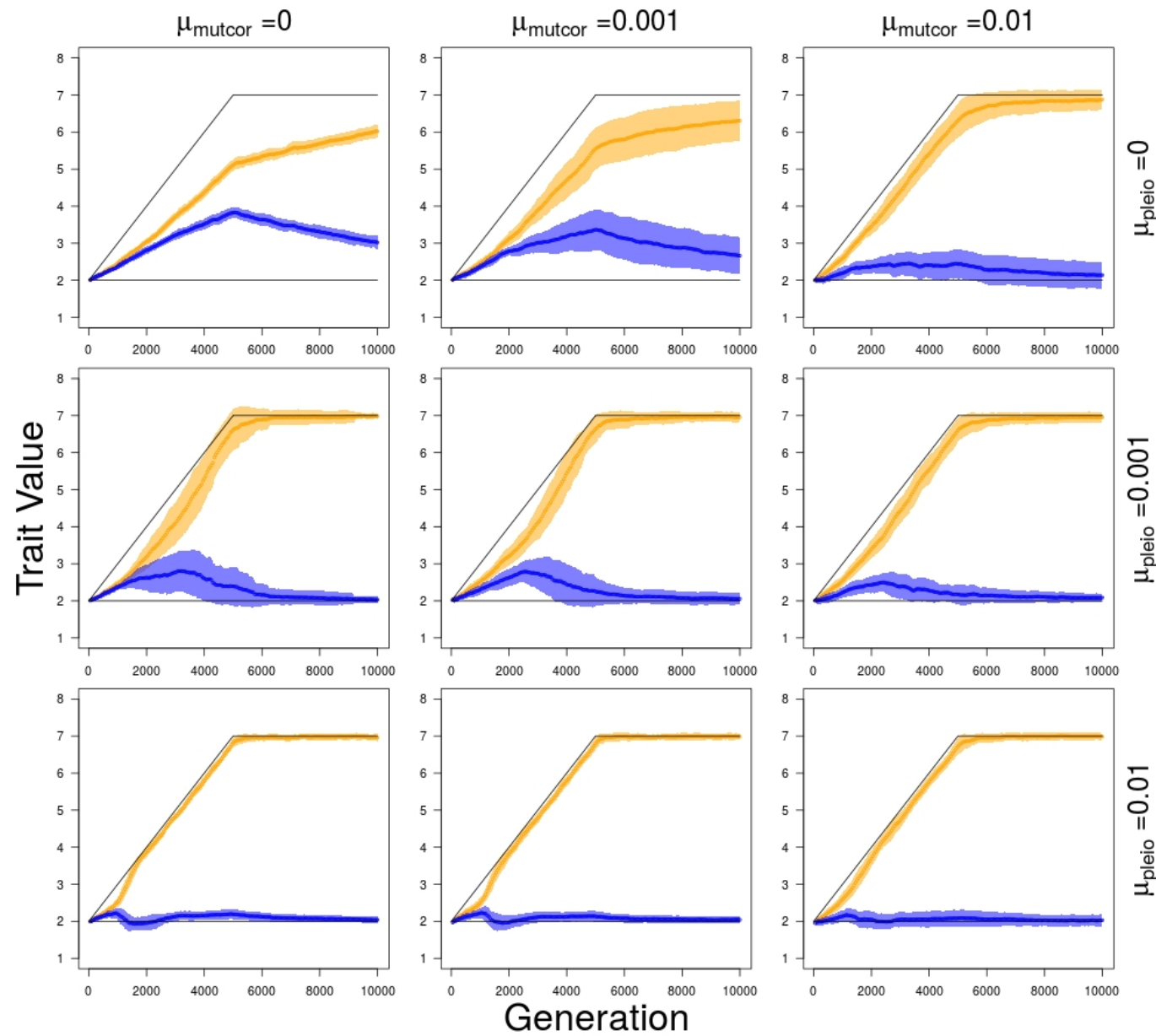

Figure 2: Trait value divergence over 5,000 generations of directional selection on traits 1 and 2 (Module 1) followed by 5,000 generations of stabilizing selection for different combinations of mutation rate in pleiotropic connections $\left(\mu_{\text {pleio }}\right)$ and mutational correlations $\left(\mu_{m u t c o r}\right)$. Orange - average values of traits 1 and 2; Blue - average values of traits 3 and 4; Black - trait value optima for modules 1 and 2. Shaded regions show standard errors of the mean for 50 replicate simulations. 


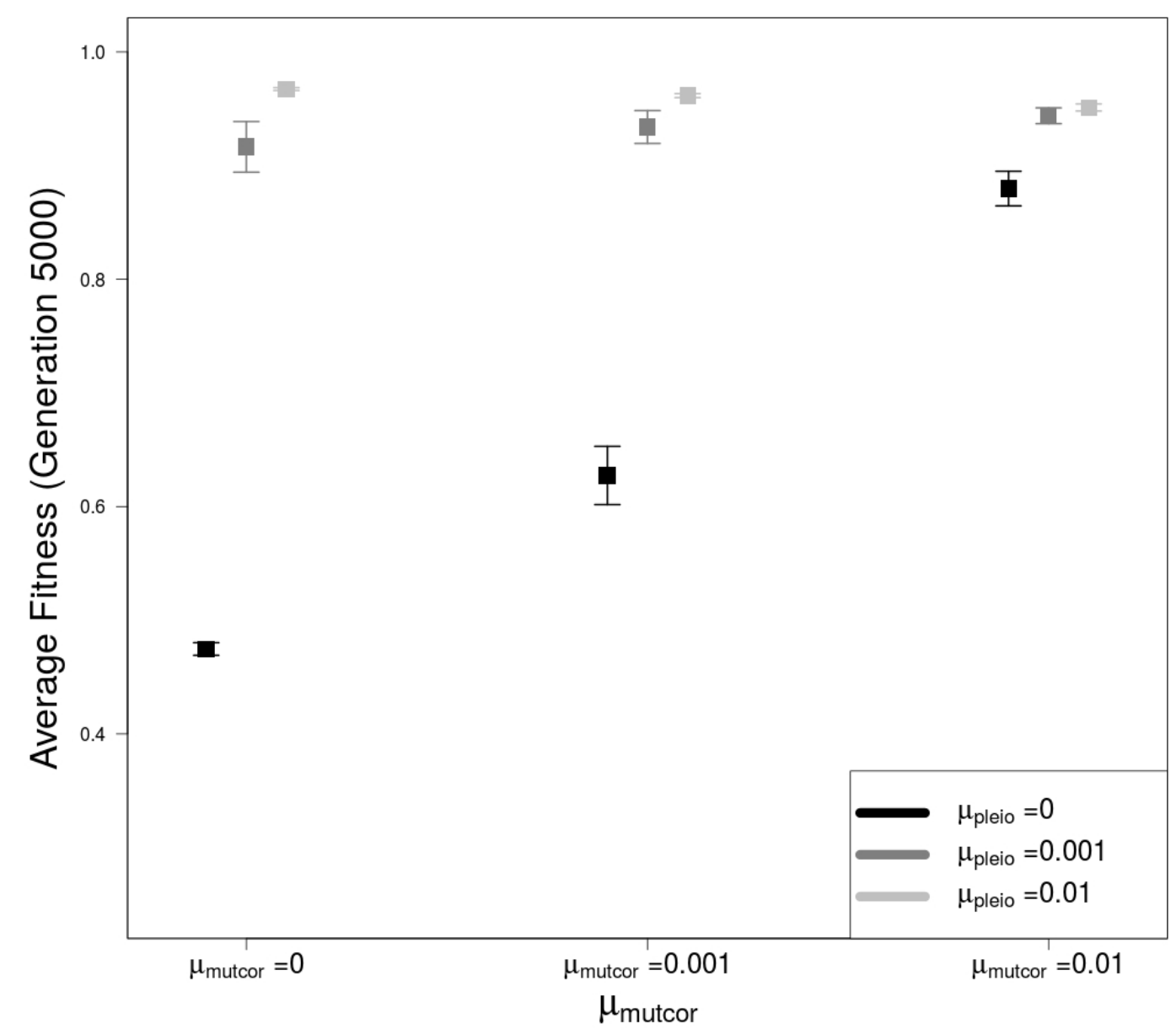

Figure 3: Average population fitness after 5,000 generations of directional selection on traits 1 and 2 (Module 1) for different combinations of mutation rate in pleiotropic connections $\left(\mu_{\text {pleio }}\right)$ and mutational correlations $\left(\mu_{\text {mutcor }}\right)$. All error bars represent standard errors of the mean for 50 replicate simulations. 


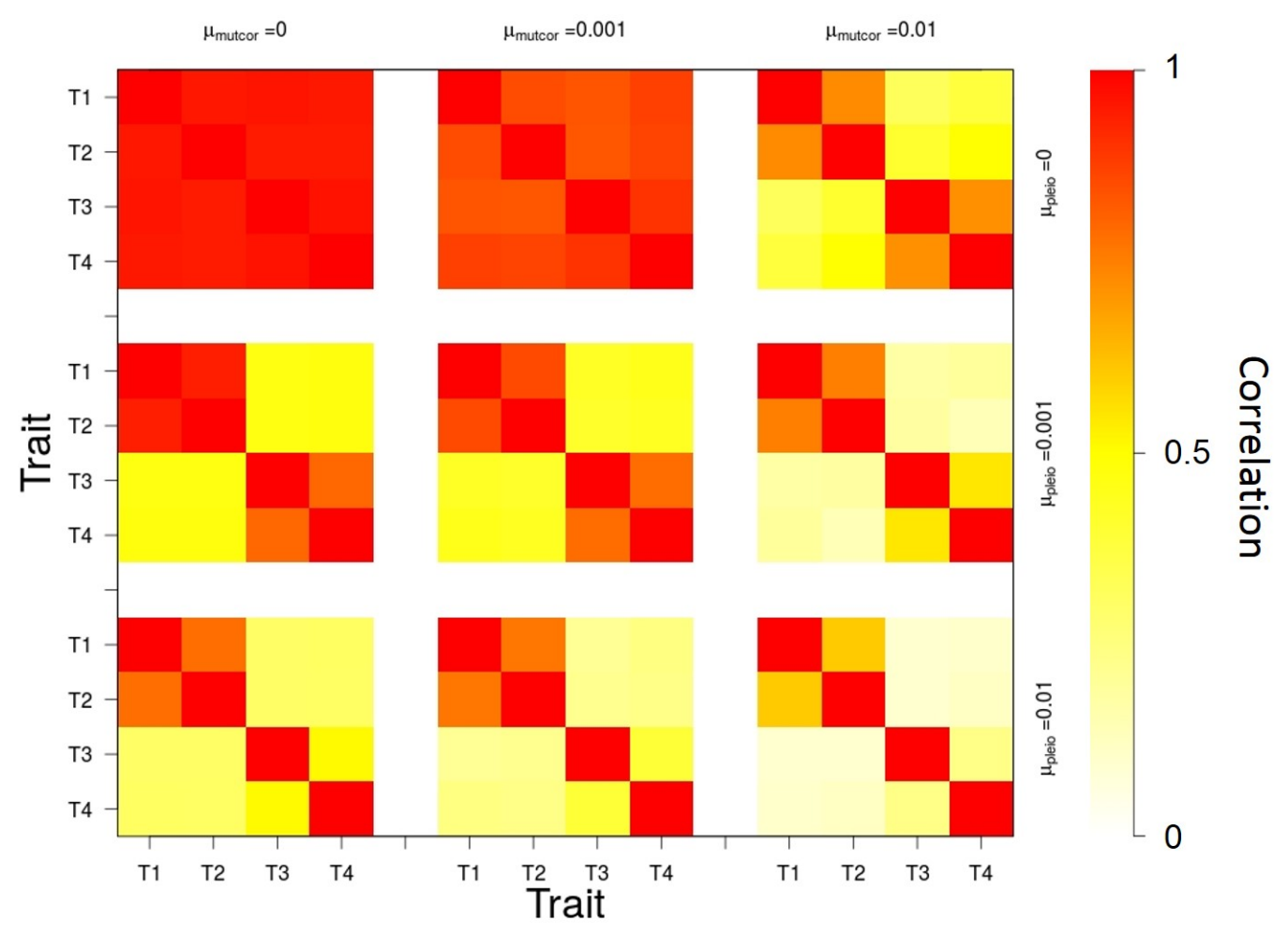

Figure 4: Genetic correlations between traits after 5,000 generations of directional selection on traits 1 and 2 (Module 1) for different combinations of mutation rate in pleiotropic connections $\left(\mu_{\text {pleio }}\right)$ and mutational correlations $\left(\mu_{\text {mutcor }}\right)$. Red - higher genetic correlation. White - no genetic correlation 
in any one individual.

When the mutation rate for pleiotropic connections $\left(\mu_{\text {pleio }}\right)$ is zero, mutational correlation evolution can still lead to divergence but this takes longer, does not diverge as fully, and therefore has lower fitness. This occurs by a general decrease in all mutational correlations between traits with the rate of decrease determined by the mutation rate of mutational correlations (Figure 6 ). When mutational correlation mutation rate is higher than the pleiotropic mutation rate then genotypic patterns do emerge where one locus is disconnected to trait 3 combines with lower mutational correlations between traits 1 and 4 or 2 and 4 , or a locus disconnected to trait 4 combines with lower mutational correlations between traits 1 and 3 or 2 and 3 (at frequencies of 0.16 and 0.10 over 50 replicates, respectively).

Effect of mutational correlation initial conditions set to zero $\left(\rho_{\mu}=0\right.$ versus $\left.\rho_{\mu}=0.99\right)$

In simulations where all mutational correlations are initialized at zero, there is little to no constraint on trait divergence despite full pleiotropic connectivity. This can be observed in trait values that follow their optima closely, leading to little reduction in fitness as optima for traits 1 and 2 diverge from traits 3 and 4 , with little evolution in mutational correlations and pleiotropic degree during divergent selection (Figure 7). There are still patterns of genetic architecture evolution as alleles with lowered pleiotropic degree still emerge in the populations, but fixation is not common nor are any allelic patterns of mutational correlations.

\section{Discussion}

\section{Pleiotropy and mutational correlation can lead to trait divergence}

Previous models of genetic architecture evolution have shown that evolution in pleiotropy and mutational correlation can influence genetic correlation between traits and therefore responses to selection, but as far as we are aware 


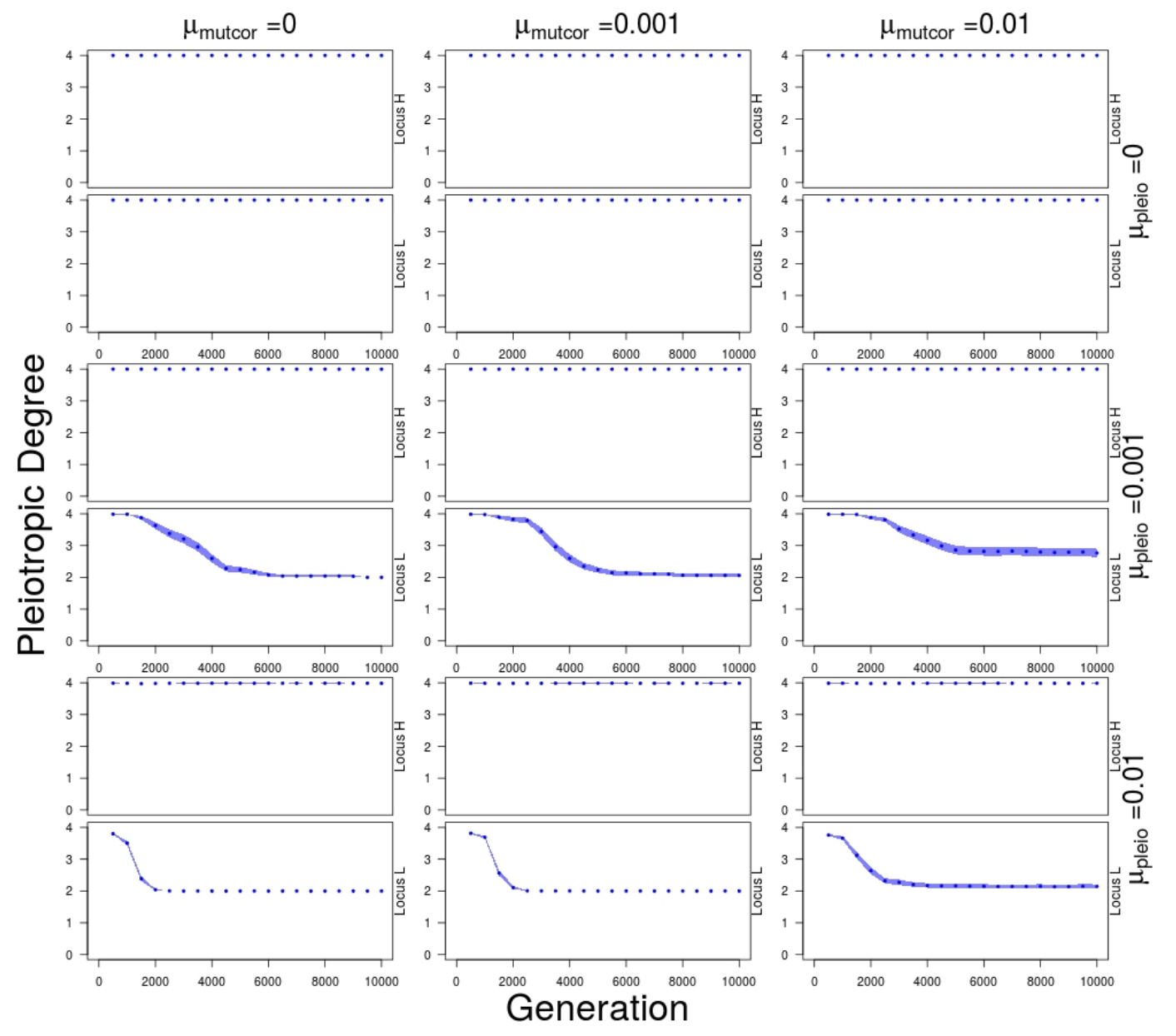

Figure 5: Average number of traits connected to each locus over 5,000 generations of directional selection on traits 1 and 2 (Module 1) followed by 5,000 generations of stabilizing selection for different combinations of mutation rate in pleiotropic connections $\left(\mu_{\text {pleio }}\right)$ and mutational correlations $\left(\mu_{\text {mutcor }}\right)$. Loci are sorted so that locus with higher pleiotropic degree (Locus H) is always shown above and lower pleiotropic degree (Locus L) shown below. Shaded regions show standard errors of the mean for 50 replicate simulations. 

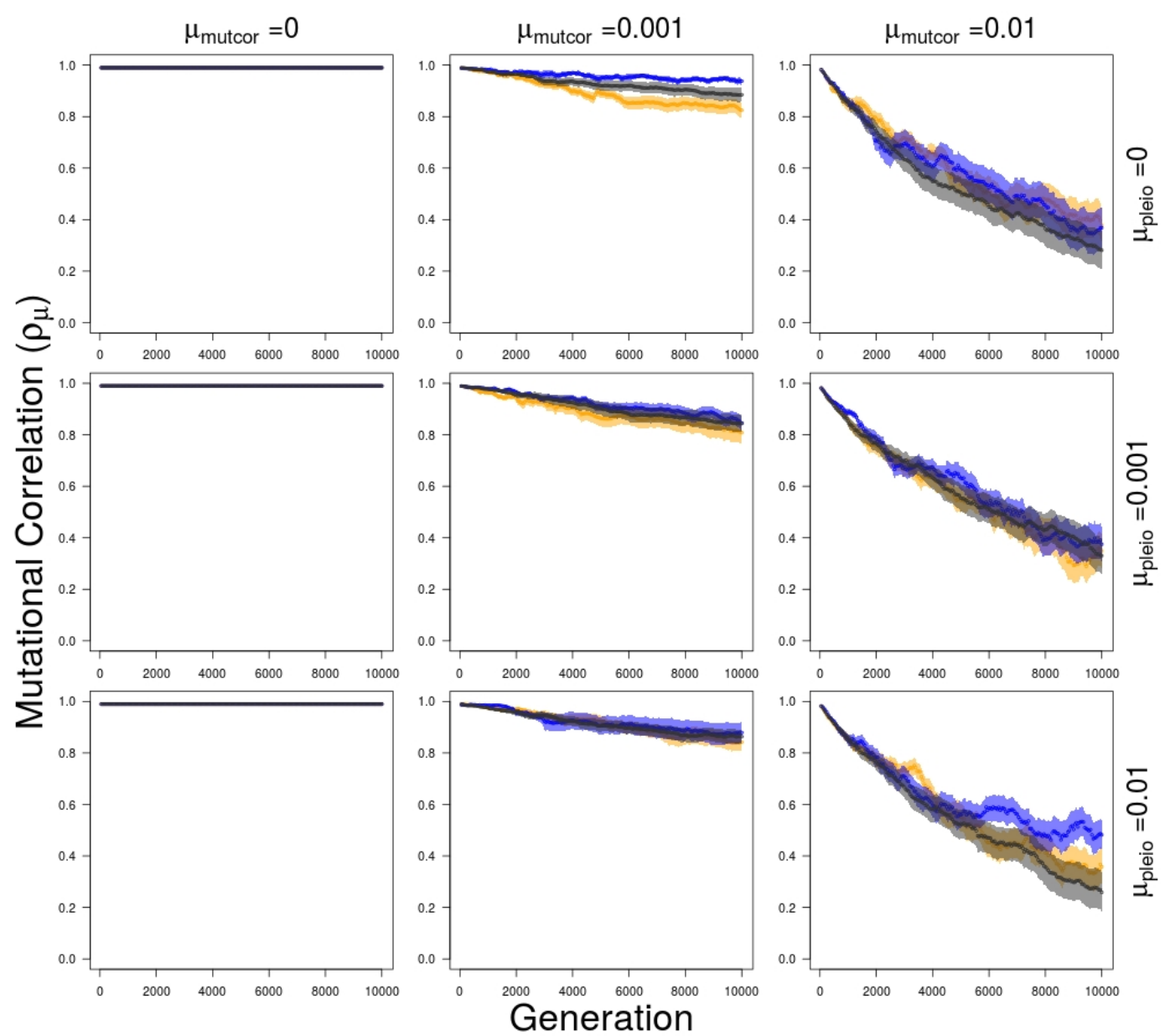

Figure 6: Average within and between module mutational correlation over 5,000 generations of directional selection on traits 1 and 2 (Module 1) followed by 5,000 generations of stabilizing selection for different combinations of mutation rate in pleiotropic connections $\left(\mu_{\text {pleio }}\right)$ and mutational correlations $\left(\mu_{\text {mutcor }}\right)$. Orange - mutational correlation between traits 1 and 2 (within Module 1); Blue - mutational correlation between traits 3 and 4 (within Module 2); Black - average mutational correlations between traits 1 and 3, 1 and 4, 2 and 3, and 2 and 4 (between Module 1 and 2). Shaded regions show standard errors of the mean for 50 replicate simulations. 

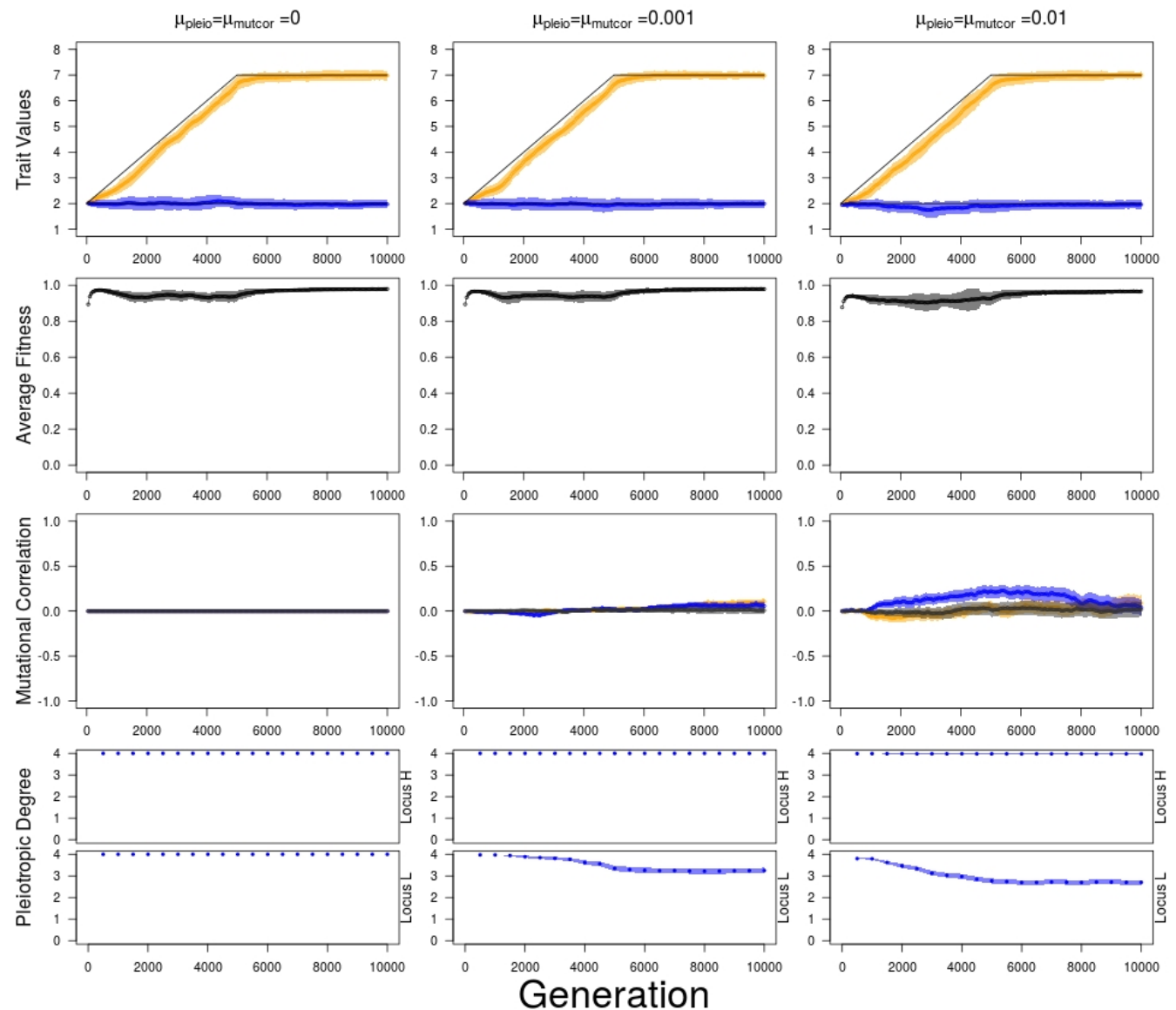

Figure 7: Average trait value, fitness, mutational correlation and pleiotropic degree, when all $\rho_{\mu}$ are initialized to 0 , over 5,000 generations of directional selection on traits 1 and 2 (Module 1) followed by 5,000 generations of stabilizing selection for different combinations of mutation rate in pleiotropic connections $\left(\mu_{\text {pleio }}\right)$ and mutational correlations $\left(\mu_{\text {mutcor }}\right)$. For pleiotropic degree, loci are sorted so that locus with higher pleiotropic degree (Locus $\mathrm{H}$ ) is always shown above and lower pleiotropic degree (Locus L) shown below. Orange trait 1 and 2 values or mutational correlation between traits 1 and 2 (Module 1); Blue trait 3 and 4 values or mutational correlation between traits 3 and 4 (Module 2); Black - average mutational correlations between traits 1 and 3,1 and 4, 2 and 3, and 2 and 4 (between Module 1 and 2). Shaded regions show standard errors of the mean for 50 replicate simulations. 
this is the first time both have been allowed to evolve in the same model to look at the relative effects of the two in constraining or facilitating evolutionary divergence. When genetic architectures are highly constraining to the divergent evolution of some traits from others, then the importance of genetic architecture evolution can clearly facilitate the rate and extent of trait divergence. Although genetic architecture may evolve through changes in pleiotropic connectivity between genes and traits, and changes in the mutational correlations between traits, the former leads to a greater release of genetic constraints and faster adaptation. A qualitative distinction exists between these two types of genetic constraints for two reasons. First, genetic constraints based on mutational correlation distributions are more difficult targets of selection compared to pleiotropic connections since distributions are cumulative phenotypes (in each generation there is only a sample of the distribution on which selection can act), whereas a single allele that differs in its pleiotropic connectivity is a phenotype on its own. Second, mutational correlations are dependent on pleiotropic connections to be effectual on traits, whereas the latter can affect the rate of adaptation regardless of mutational correlation (Baatz and Wagner, 1997; Chebib and Guillaume, 2017).

The results of this study corroborate results from previous models of pleiotropic evolution. We observe that divergent selection in the form of the corridor model leads to modular genetic architecture with greater genetic correlations between traits within modules and lower correlations between modules. This was also the case in both Melo and Marroig (2015) and Pavlicev et al. (2011) under the corridor model. They observed higher genetic correlations in modules under directional selection than under stabilizing selection which can also be seen in the patterns of genetic correlation we observed where traits 1 and 2 (traits under directional selection) had higher correlations between them than between traits 3 and 4 (traits under stabilizing selection). Unfortunately, it is unclear whether patterns of partial modular pleiotropy were responsible for the emergence of modularity in Melo 
and Marroig (2015) as was found in our study. Jones et al. (2007) found "extreme" variation among replicates in the average mutational correlation observed when it was capable of evolving as was observed in our study (as well as when simulations were run with the same parameter values as the Jones et al. (2007) study; Supplemental Figure S1). The same "indeterminacy in the evolution" of mutational correlation is likely responsible for the variation among replicates due to an unstable equilibrium in the adaptive landscape (Lande (1980); Zhang and Hill (2002); Jones et al. (2007); Supplemental Figure S2).

\section{Patterns of pleiotropy}

What explains the emergence of one dominant genotype that has one locus losing its connections to traits 3 and 4, and the other locus maintaining full pleiotropy? When mutational correlations are strong, modularization should arise so that mutational effects can increase traits 1 and 2 values without also increasing traits 3 and 4, (especially when stabilizing selection is strong compared to directional selection). Had stabilizing selection been weaker and/or directional selection been much stronger, then more loci affecting the traits would have increased the proportion of advantageous mutations allowing for divergence (Hansen, 2003). For the same reason, we don't observe complete modularization with one locus only connected to traits 1 and 2 , and the other only connected to traits 3 and 4 . With both loci contributing to traits 1 and 2, there is more mutational input from which larger effects to increase their values may appear, giving support to the idea that intermediate levels of integration will maximize evolvability when pleiotropic effects are all positive (Hansen, 2003). Also, since we were interested in the evolution of genetic architecture with trait divergence after gene duplication we started our simulations with a highly constrained, monomorphic population. This makes evolution in our model dependent on mutation and as traits diverged, the negative effects of pleiotropy increased, leading to modularization in the genetic architecture. But when mutation rates are high, and 
selection acts primarily on standing genetic variation (from many mutations of small effects), pleiotropy is less constraining and integrated genetic architectures (both loci affecting all traits in our simulation) are more evolvable (as the analytical model of Pavlicev and Hansen (2011) shows). Therefore, if variation in pleiotropic connections and mutational correlations exist within duplicated loci in a population prior to divergent selection we may expect that many possible combinations of alleles that allow for increased variation and reduced covariation between traits could also exist. In that scenario, modularization may not be associated with trait divergence. In our simulations there is a glimpse of evolvability less modularity when mutational correlation is initialized to zero and traits are able to diverge following their respective optima closely without a decrease in fitness.

The results we obtain in this study are also related to work done on the evolutionary fate of duplicated, pleiotropic genes (Ohno, 1970; Hahn, 2009; Innan and Kondrashov, 2010; Guillaume and Otto, 2012). Previous models describe the conditions under which both genes remained fully pleiotropic which is expected to be favorable when there is selection for increased dosage as we had for traits 1 and 2 (Ohno, 1970). There is some empirical evidence of this in ribosomal RNA, histone genes, as well as amylase genes in humans with high starch diets (Zhang, 2003; Perry et al., 2007; Qian et al., 2010). Other models describe when one or both genes lose their connection to some traits, known as subfunctionalization, if there is a relaxation of selection after duplication (Force et al., 1999; Lynch and Force, 2000). Their is also plenty of empirical evidence that this has occurred in vertebrate limb evolution, as discussed in the introduction, as well as pathway specialization in plants (Bomblies and Doebley, 2006; Des Marais and Rausher, 2008). The difference in our results compared to models with selection for increased dosage is that in our model there is only selection for higher values in traits 1 and 2 , whereas selection for increased values in all four traits is expected to maintain all pleiotropic connections. The difference compared to neutral 
models where subfunctionalization is the result is that in our model there is no relaxation of selection due to duplication and redundancy. The maintenance of pleiotropy in one gene and subfunctionalization in the other (the most common outcome in our simulations) is predicted to occur under specific conditions where there is asymmetry in either the fitness effects of traits or in the expression levels of genes but this prediction was the result of a model where there is competitive allocation of a gene's resources to the functions of one trait or another (as opposed to a trade-off at the fitness level in our model (Guillaume and Otto, 2012)). Additionally, mutations in this competitive allocation model can change the pleiotropic degree of genes in increments along a continuum (instead of connected or disconnected), so it is difficult to determine whether these similar observations result through the same mechanisms.

\section{Conclusion}

Both pleiotropic connections and mutational correlations can constrain the divergence of traits under directional selection from traits under stabilizing selection but when both can evolve trait divergence occurs because pleiotropic connections are broken between loci and traits under stabilizing selection. This is the result of pleiotropic connectivity being an easier target of selection than a distribution of mutational effects. The most commonly observed genotype includes one locus that maintains connectivity to both traits under directional selection and both traits under stabilizing selection, and the other locus losing its connection to the traits under stabilizing selection (subfunctionalization). The subfunctionalization of one locus allows it to contribute to increasingly divergent trait values in the traits under directional selection without changing the trait values of the other traits which leads to separate genetic modules. These results indicate that partial subfunctionalization is sufficient to allow genetic modularization and the divergence of traits with little to no loss of average fitness. 


\section{Supplemental}

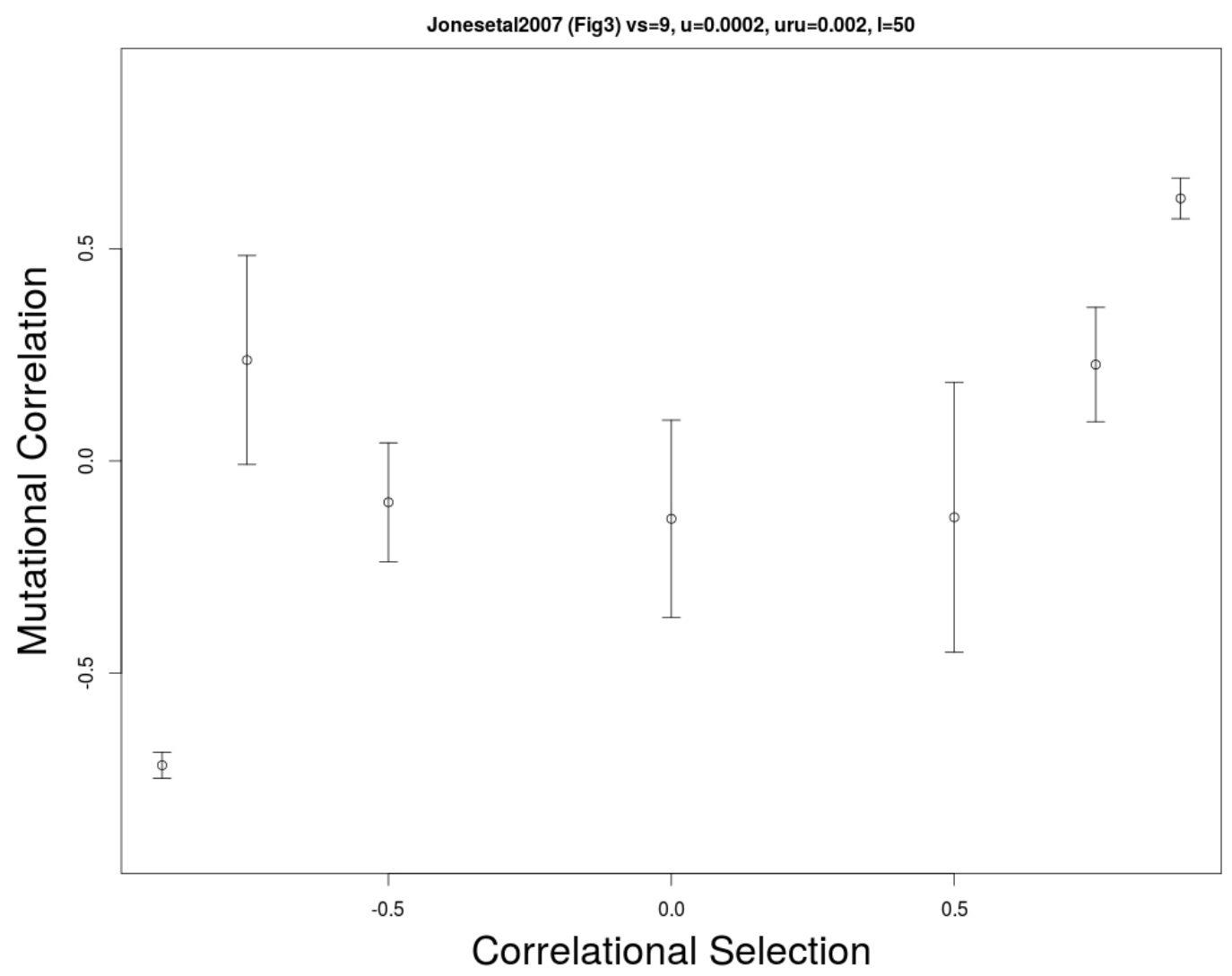

Figure S1: Average mutational correlation $\rho_{\mu}$ values for different values of correlational selection $\rho_{\omega}$. Parameter values were chosen to match those used in Jones et al. 2007 wherever possible and averages were taken over values from every five generations after burn-in between generation 10,000 and 20,000. Number of loci $=50$, Number of traits $=$ $2, N=2372, \omega^{2}=9, \mu=0.0002, \mu_{\text {mutcor }}=0.002$, and $\alpha^{2}=0.05$. 


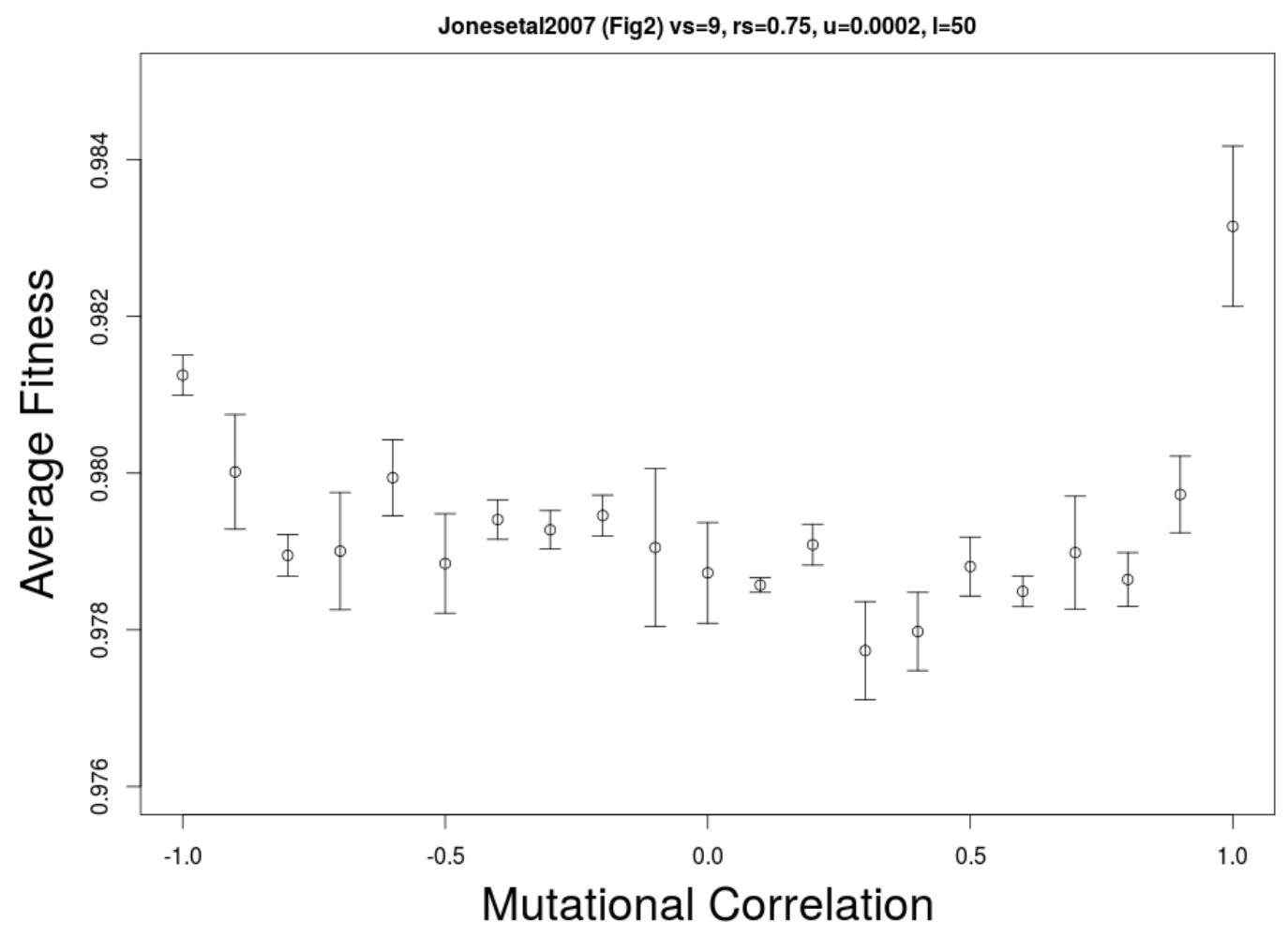

Figure S2: Average fitness for different values of mutational correlation (static). Parameter values were chosen to match those used in Jones et al. 2007 wherever possible and averages were taken over values from every five generations after burn-in between generation 10,000 and 15,000. Number of loci $=50$, Number of traits $=2, N=2372, \omega^{2}=9, \rho_{\omega}=0.75, \mu$ $=0.0002$, and $\alpha^{2}=0.05$. 


\section{References}

Arnold, S. J. (1992). Constraints on phenotypic evolution. The American Naturalist, 140:S85-S107.

Baatz, M. and Wagner, G. P. (1997). Adaptive inertia caused by hidden pleiotropic effects. Theoretical Population Biology, 51(1):49-66.

Bomblies, K. and Doebley, J. F. (2006). Pleiotropic effects of the duplicate maize floricaula/leafy genes zfl1 and zfl2 on traits under selection during maize domestication. Genetics, 172(1):519-531.

Bürger, R. (1986). Constraints for the evolution of functionally coupled characters: a nonlinear analysis of a phenotypic model. Evolution, 40(1):182193.

Chebib, J. and Guillaume, F. (2017). What affects the predictability of evolutionary constraints using a g-matrix? the relative effects of modular pleiotropy and mutational correlation. Evolution.

Crow, J. F. and Kimura, M. (1964). The theory of genetic loads. Proc. XI Int. Congr. Genetics, 2:495-505.

Des Marais, D. L. and Rausher, M. D. (2008). Escape from adaptive conflict after duplication in an anthocyanin pathway gene. Nature, 454(7205):762.

Force, A., Lynch, M., Pickett, F. B., Amores, A., Yan, Y.-l., and Postlethwait, J. (1999). Preservation of duplicate genes by complementary, degenerative mutations. Genetics, 151(4):1531-1545.

Graham, A. and McGonnell, I. (1999). Limb development: Farewell to arms. Current biology, 9(10):R368-R370.

Guillaume, F. and Otto, S. P. (2012). Gene functional trade-offs and the evolution of pleiotropy. Genetics, 192:1389-1409. 
Guillaume, F. and Rougemont, J. (2006). Nemo:an evolutionary and population genetics programming framework. Bioinformatics, 22:2556-2557.

Hahn, M. W. (2009). Distinguishing among evolutionary models for the maintenance of gene duplicates. Journal of Heredity, 100(5):605-617.

Hansen, T. F. (2003). Is modularity necessary for evolvability?: Remarks on the relationship between pleiotropy and evolvability. Biosystems, 69(2):8394.

Hansen, T. F. (2006). The evolution of genetic architecture. Ann. Rev. Ecol. Evol. Syst., 37:123-157.

Innan, H. and Kondrashov, F. (2010). The evolution of gene duplications: classifying and distinguishing between models. Nature Reviews Genetics, $11(2): 97$.

Jones, A. G., Arnold, S. J., and Bürger, R. (2003). Stability of the g-matrix in a population experiencing pleiotropic mutation, stabilizing selection, and genetic drift. Evolution, 57:1747-1760.

Jones, A. G., Arnold, S. J., and Bürger, R. (2007). The mutation matrix and the evolution of evolvability. Evolution, 61(4):727-745.

Jones, A. G., Bürger, R., and Arnold, S. J. (2014). Epistasis and natural selection shape the mutational architecture of complex traits. Nat. Comm., 5:3709-3718.

Kenney-Hunt, J. P., Wang, B., Norgard, E. A., Fawcett, G., Falk, D., Pletscher, L. S., Jarvis, J. P., Roseman, C., Wolf, J., and Cheverud, J. M. (2008). Pleiotropic patterns of quantitative trait loci for 70 murine skeletal traits. Genetics, 178(4):2275-2288.

Lande, R. (1979). Quantitative genetic analysis of multivariate evolution, applied to brain:body size allometry. Evolution, 33:402416. 
Lande, R. (1980). The genetic covariance between characters maintained by pleiotropic mutations. Genetics, 94(1):203-215.

Lynch, M. and Force, A. (2000). The probability of duplicate gene preservation by subfunctionalization. Genetics, 154(1):459-473.

Melo, D. and Marroig, G. (2015). Directional selection can drive the evolution of modularity in complex traits. Proc. Natl. Acad. Sci. U.S.A., 112:470475.

Melo, D., Porto, A., Cheverud, J. M., and Marroig, G. (2016). Modularity: genes, development, and evolution. Annual review of ecology, evolution, and systematics, 47:463-486.

Minguillon, C., Gibson-Brown, J. J., and Logan, M. P. (2009). Tbx4/5 gene duplication and the origin of vertebrate paired appendages. Proceedings of the National Academy of Sciences, pages pnas-0910153106.

Muller, H. J. (1936). Bar duplication. Science, 83(2161):528-530.

Ohno, S. (1970). Evolution by gene duplication springer. New York.

Orr, H. A. (2000). Adaptation and the cost of complexity. Evolution, $54(1): 13-20$.

Pavlicev, M., Cheverud, J. M., and Wagner, G. P. (2011). Evolution of adaptive phenotypic variation patterns by direct selection for evolvability. Proceedings of the Royal Society of London B: Biological Sciences, 11:rspb20102113.

Pavlicev, M. and Hansen, T. F. (2011). Genotype-phenotype maps maximizing evolvability: Modularity revisited. Evolutionary Biology, 38(4):371389. 
Perry, G. H., Dominy, N. J., Claw, K. G., Lee, A. S., Fiegler, H., Redon, R., Werner, J., Villanea, F. A., Mountain, J. L., Misra, R., et al. (2007). Diet and the evolution of human amylase gene copy number variation. Nature genetics, 39(10):1256.

Petit, F., Sears, K. E., and Ahituv, N. (2017). Limb development: a paradigm of gene regulation. Nature Reviews Genetics, 18(4):245.

Qian, W., Liao, B.-Y., Chang, A. Y.-F., and Zhang, J. (2010). Maintenance of duplicate genes and their functional redundancy by reduced expression. Trends in Genetics, 26(10):425-430.

Rastogi, S. and Liberles, D. A. (2005). Subfunctionalization of duplicated genes as a transition state to neofunctionalization. BMC evolutionary biology, 5(1):28.

Rueffler, C., Hermisson, J., and Wagner, G. P. (2012). Evolution of functional specialization and division of labor. Proceedings of the National Academy of Sciences, 109(6):E326-E335.

Smith, S. D. (2016). Pleiotropy and the evolution of floral integration. New Phytologist, 209(1):80-85.

Stern, D. L. (2000). Perspective: evolutionary developmental biology and the problem of variation. Evolution, 54(4):1079-1091.

Wagner, G. (1984). Coevolution of functionally constrained characters: prerequisites for adaptive versatility. BioSystems, 17(1):51-55.

Wagner, G. P. (1989). Multivariate mutation-selection balance with constrained pleiotropic effects. Genetics, 122(1):223-234.

Wagner, G. P. and Altenberg, L. (1996). Perspective: complex adaptations and the evolution of evolvability. Evolution, 50(3):967-976. 
${ }_{434}$ Wang, Z., Liao, B. Y., and Zhang, J. Z. (2010). Genomic patterns of 435 pleiotropy and the evolution of complexity. Proc. Natl. Acad. Sci. U.S.A., $436 \quad 107: 18034-18039$.

${ }_{437}$ Welch, J. J. and Waxman, D. (2003). Modularity and the cost of complexity. ${ }_{438}$ Evolution, 57(8):1723-1734.

442

443

Zhang, J. (2003). Evolution by gene duplication: an update. Trends in ecology \& evolution, 18(6):292-298.

Zhang, X. S. and Hill, W. G. (2002). Joint effects of pleiotropic selection and stabilizing selection on the maintenance of quantitative genetic variation at mutation-selection balance. Genetics. 